Rakenteiden Mekaniikka (Journal of Structural Mechanics)

Vol. 50, No 3, 2017, pp. $127-130$

https://rakenteidenmekaniikka.journal.fi/index

https://doi.org/10.23998/rm.65104

(c) The author(s) 2017.

Open access under CC BY-SA 4.0 license.

\title{
Evaluation of some harmonic load models for the vibration analysis of footbridges
}

\author{
Antti H. Niemi ${ }^{1}$, Jani Koskela and Filip Fedorik
}

Summary. We analyze and compare different dynamic load models used for the verification of vibration serviceability of footbridges. The considered models predict the acceleration response of the bridge for random streams of pedestrians as well as for deterministic group of walking or running pedestrians. The analysis is carried out in the context of a steel-concrete composite bridge model. Both moving and stationary loads are studied.

Key words: footbridge, vibration serviceability, human induced vibrations, structural dynamics

Received 29 June 2017. Accepted 7 July 2017. Published online 21 August 2017

\section{Introduction}

The current architectural, structural and economical trends for pedestrian bridges lead towards light and slender structures, where dynamic behaviour plays a big role in terms of stability and comfort of users. The European design standard [1] requires that appropriate dynamic models and comfort criteria should be defined but leaves a more detailed specification to the designer and/or national annexes. Consequently, different guidelines and load models have been developed and analyzed, see e.g. [2, 3]. We analyze and compare here three such load models in context of a bridge model.

\section{Mathematical model}

The starting point of our study is a conventional structural model for a single-span footbridge based on the Euler-Bernoulli beam theory. The governing differential equation can be written in terms of the beam deflection $w$ as

$$
E I \frac{\partial^{4} w}{\partial x^{4}}(x, t)+c \frac{\partial w}{\partial t}(x, t)+\rho A \frac{\partial^{2} w}{\partial t^{2}}(x, t)=f(x, t), \quad 0<x<L, t>0,
$$

where $E$ is Young's modulus of the material and $I$ and $A$ are the moment of inertia and area of the beam cross section, respectively. The parameter $c$ is the viscous damping parameter and $\rho$ stands for the mass density of the material. All parameters are assumed

${ }^{1}$ Corresponding author. antti.niemi@oulu.fi

This work has been supported by the Finnish Transport Agency and Oulun rakennustekniikan säätiö. 
to be constant over the interval $(0, L)$, where $L$ is the length of the bridge. The external load is represented by the function $f(x, t)$. It should be noted that the rotary inertia and transverse shear stiffness are ignored in this model.

The equation of motion can be decoupled in space and time by using the separation of variables

$$
w(x, t)=z(t) \phi(x) .
$$

By utilizing the principle of virtual displacements or the method of weighted residuals, the equation of motion (1) can be transformed to the form

$$
\tilde{k} z(t)+\tilde{c} z^{\prime}(t)+\tilde{m} z^{\prime \prime}(t)=\tilde{f}(t)
$$

For simply supported or hinged ends, the first bending mode is $\phi(x)=\sin (\pi x / L)$ and the corresponding generalized stiffness, mass and damping coefficients are

$$
\begin{aligned}
\tilde{k} & =\int_{0}^{L} E I\left[\phi^{\prime \prime}(x)\right]^{2} \mathrm{~d} x=E I \frac{\pi^{4}}{2 L^{3}}, \\
\tilde{c} & =\int_{0}^{L} c[\phi(x)]^{2} \mathrm{~d} x=\frac{c L}{2}, \\
\tilde{m} & =\int_{0}^{L} \rho A[\phi(x)]^{2} \mathrm{~d} x=\frac{M}{2},
\end{aligned}
$$

where $M=\rho A L$ is the total mass of the bridge. Finally, the generalized load is defined as

$$
\tilde{f}(t)=\int_{0}^{L} f(x, t) \phi(x) \mathrm{d} x .
$$

Division of Eq. (2) by the modal mass $\tilde{m}$ yields the customary form of a damped harmonic oscillator

$$
z^{\prime \prime}(t)+2 \zeta \omega z^{\prime}(t)+\omega^{2} z(t)=\frac{\tilde{f}(t)}{\tilde{m}}
$$

where

$$
\omega=\sqrt{\frac{\tilde{k}}{\tilde{m}}}=\pi^{2} \sqrt{\frac{E I}{M L^{3}}}
$$

is the natural frequency of the bridge and

$$
\zeta=\frac{\tilde{c}}{2 \sqrt{\tilde{m} \tilde{k}}}
$$

is the dimensionless damping ratio.

\section{Load models}

As an illustration, we consider three different types of harmonic loads of the form

$$
f_{i}(x, t)=p_{i}(x, t) \sin (\omega t), \quad i=1,2,3 .
$$

The first model is associated to the so called pedestrian stream model, where the random load due to a stream of random pedestrians is replaced by an equivalent uniformly distributed load $p_{1}$ over the bridge surface area. The second load consists of a group of 
walkers/joggers with a weight $P_{2}$ moving with a constant velocity $v$ across the bridge. Finally, in the third case the load $P_{3}$ is assumed to act at the center of the bridge at $x=L / 2$, where the maximum amplitude of the first bending mode occurs. This kind of models have been advocated in [2] and in the references therein.

Referring to the assumed structural model, the load functions for the above load cases are

$$
\begin{aligned}
& f_{1}(x, t)=p_{1} \sin (\omega t), \\
& f_{2}(x, t)=P_{2} \delta(x-v t) \sin (\omega t), \\
& f_{3}(x, t)=P_{3} \delta(x-L / 2) \sin (\omega t),
\end{aligned}
$$

where $\delta(\cdot)$ stands for the Dirac delta function. The corresponding generalized loads according to Eq. (3) are then

$$
\begin{aligned}
& \tilde{f}_{1}(t)=\frac{2}{\pi} L \cdot p_{1} \sin (\omega t), \\
& \tilde{f}_{2}(t)=P_{2} \sin \left(\frac{\pi t}{T}\right) \sin (\omega t), \\
& \tilde{f}_{3}(t)=P_{3} \sin (\omega t)
\end{aligned}
$$

where $T=L / v$ is the time it takes for the group to cross the bridge in the second load case.

\section{Numerical results and conclusions}

We consider now a steel-concrete composite single-span bridge model which has been verified in the ultimate limit state. The bridge properties are as follows:

- Length and width: $L=33 \mathrm{~m} \& H=3 \mathrm{~m}$

- Total mass: $M=105417 \mathrm{~kg}$

- Bending rigidity: $E I=7253400 \mathrm{kN} \cdot \mathrm{m}^{2}$

- Natural frequency: $f=\frac{\omega}{2 \pi}=2.174 \mathrm{~Hz}$

- Assumed damping ratio: $\zeta=0.3 \%$

In the first loading case, we assume a pedestrian density of $d=0.2 \mathrm{~m}^{-2}$. According to the pedestrian stream model, see [2], the corresponding equivalent number of pedestrians uniformly distributed on the loaded surface is

$$
n^{\prime}=10.8 \frac{\sqrt{\zeta d L H}}{L H}=0.02659 \mathrm{~m}^{-2}
$$

In this model, the vertical load is assumed as $P_{\mathrm{w}}=280 \mathrm{~N}$ and the reduction factor corresponding to the natural frequency is calculated as $\psi_{1}=0.632$ based on Table 4-8 of [2]. The distributed load over the beam length becomes $p_{1}=P_{\mathrm{w}} \cdot \psi_{1} \cdot n^{\prime} \cdot H=14.115 \mathrm{~N} / \mathrm{m}$.

In the second loading case with a moving load, we assume a pair of joggers $(n=2)$ running with a constant velocity of $v=3 \mathrm{~m} / \mathrm{s}$. The vertical load per jogger is $P_{\mathrm{j}}=1250 \mathrm{~N}$ and the frequency-dependent reduction factor is determined here as $\psi_{2}=0.931$ based on Table 9-2 of [2] yielding a load amplitude $P_{2}=n \cdot P_{\mathrm{j}} \cdot \psi_{2}=2.328 \mathrm{kN}$. 


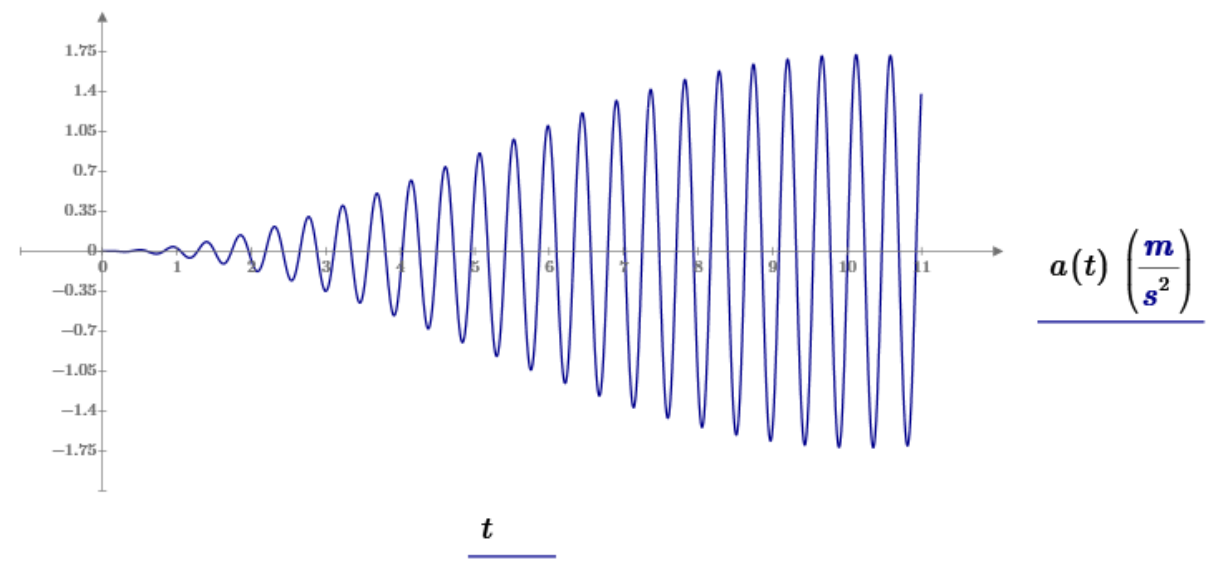

Figure 1. Acceleration response of the model bridge for a moving load.

In the third loading case with a stationary load at the center, we reduce the number of joggers as $n^{\prime}=\sqrt{n}$ so that $P_{3}=n^{\prime} \cdot P_{\mathrm{j}} \cdot \psi_{2}=1.646 \mathrm{kN}$.

The maximum acceleration in the first and third cases can be calculated as based on the analytic solution of the damped harmonic oscillator (4):

$$
a_{\max , 1}=\frac{2}{\pi} \frac{p_{1} L}{\zeta M}=0.938 \frac{\mathrm{m}}{\mathrm{s}^{2}} \quad \& \quad a_{\max , 3}=\frac{P_{3}}{\zeta M}=5.204 \frac{\mathrm{m}}{\mathrm{s}^{2}}
$$

In the second case, the acceleration response was evaluated numerically and is shown in Fig. 1. The corresponding maximum acceleration is estimated to be around

$$
a_{\max , 2}=1.7 \frac{\mathrm{m}}{\mathrm{s}^{2}} \text {. }
$$

We observe that uncomfortable acceleration levels $\left(\geq 0.7 \frac{\mathrm{m}}{\mathrm{s}^{2}}\right)$ are predicted for all loading cases. We conclude that the jogger loading case is very demanding and the load model where the load is fixed at the center yields very high accelerations as compared to the corresponding moving load.

\section{References}

[1] EN 1991-2. Eurocode 1: Actions on structures - Part 2: Traffic loads on bridges. 2003.

[2] C. Heinemeyer et al. Design of Lightweight Footbridges for Human Induced Vibrations JRC Scientific and Technical Reports, 2009.

[3] K. van Nimmen, G. Lombaert, G. De Roeck, and P. van den Broeck. Vibration serviceability of footbridges: Evaluation of the current codes of practice. Engineering Structures, 59:448-461, 2014. https:/doi.org/10.1016/j.engstruct.2013.11.006

Antti H. Niemi, Filip Fedorik

University of Oulu

P.O.Box 4200, FI-90014 Oulu

antti.niemi@oulu.fi, filip.fedorik@oulu.fi

Jani Koskela

Pöyry Finland Oy

Elektroniikkatie 13, 90590 Oulu

jani.koskela@poyry.fi 